\title{
Fish hook injury to the eye and its management
}

\author{
Sangeetha Jeganathan, Suneetha Nithyanandam, Reji K. Thomas, \\ Shubashree Karat \\ Department of Ophthalmology, St Johns Medical College and Hospital, \\ Bangalore, India
}

\begin{abstract}
The most common cause of unilateral blindness in paediatric age groups, especially in developing countries, is ocular trauma. Trauma to the eye is very debilitating to the patient; hence, managing various types of penetrating and blunt injury to the eye provide a challenge to the ophthalmologist. It is simply preventable by the supervision of the parents and caregivers. We will be discussing one such case and our experience in dealing with a fish hook injury to the eye in a 12-year-old boy-the challenges we faced and the precautions we took to give a good visual outcome.
\end{abstract}

Keywords: advance-and-cut technique, fish hook, open globe injury, visual outcome

\section{Introduction}

Ocular trauma, although not an everyday encounter for many ophthalmologists, is a serious problem for our health system and economy. ${ }^{1,2}$ Ocular injuries can occur in any setting, including recreational and sports-related activities, the workplace, the home, rural agricultural settings, motor vehicle accidents and intentional altercations. The personal impact of ocular injury is difficult to define, although the lifestyle of the affected individual may be permanently altered. In addition to the visual concerns, ocular trauma levies a tremendous financial penalty in terms of both direct and indirect costs. During the last several decades, the prognosis for patients with ocular injuries, especially those with open globe injuries, has significantly improved. This has been attributed to the advent of enhanced microsurgical techniques and instrumentation, along with an improved understanding of the pathophysiological mechanisms of ocular trauma, as well as the availability of new antibiotics which can achieve good intraocular concentrations. Eye injuries can be greatly reduced by promoting the concept of prevention by the ophthalmologist. To do an effective job of prevention, the ophthalmologist must have knowledge of the eye injury potential, a particular patient may experience and the proper protective devices that are available to prevent the same.

Correspondence: Dr. Sangeetha Jeganathan,

Department of Ophthalmology, St Johns Medical College and Hospital, Sarjapur Main

Rd, John Nagar, Koramangala, Bengaluru, Karnataka 560034, Bangalore, India.

E-mail: drjsangs81@gmail.com 


\section{Case report}

A 12-year-old boy presented to us at the emergency with history of foreign body in the left eye while fishing. He complained of minimal discomfort and diminution of vision.

On examination, his visual acuity was $20 / 20$ in right eye and finger counting close to face in left eye with accurate projection of rays. Anterior segment of the left eye showed a fish hook passing through the limbus with the entry point at 9 o'clock and exit point at 6 o'clock position as shown in Figure 1. There was associated subconjunctival haemorrhage in the area. Siedel's test was negative. Cornea was clear without any tear and anterior chamber was well formed with no signs of inflammation or hyphaema. Pupil was brisk reacting briskly to direct and consensual light reflex. Lens was clear and fundus was normal. The intraocular pressure (IOP) at presentation was $16 \mathrm{mmHg}$. Computed tomography (CT) orbit showed a fish hook passing at the limbus through the uveal tissue and with the barbed end at the exit as shown in Figure 2.

We got a clearance from the anaesthetist and took up the child for removal of the fish hook and primary repair under general anaesthesia. A peritomy was done and wound explored, and in view of the arrowhead-shaped hook, it was decided to pull the hook out through the exit wound to prevent ripping up of the tissues by the arrow head. As the hook was lodged in the ciliary body zone,



Fig. 1. Photograph showing the barbed fish hook penetrating the limbus at 9 o'clock position and exit point seen at 6 o'clock position. 


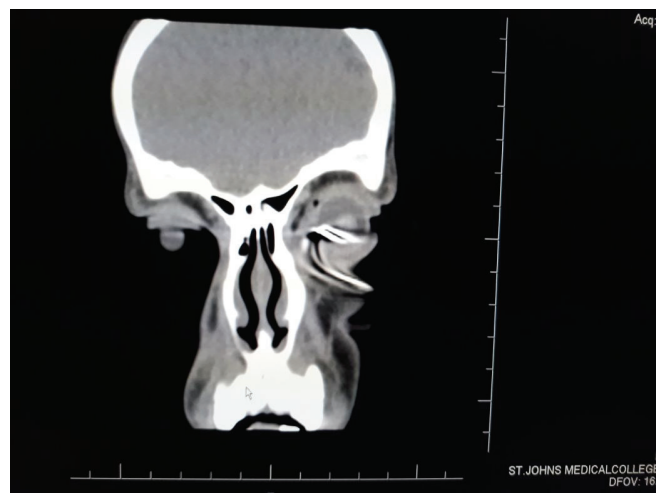

Fig. 2. CT orbit showing the end on view of the fish hook embedded in the eye.

cryotherapy was done around the hook to prevent and minimize the bleeding during the removal of the hook. Following cryotherapy, the metallic knob at the entry site was cut using bone cutter (Fig. 3), then the hook was slowly pushed out of the exit site; however, this was not fruitful as the hook was deeply imbedded and there was bleeding during the manoeuvre. Hence, using a 15-number BP blade, we made an incision along the shank of the hook at the inferior limbus and extracted the hook. The wound was sutured with 10-0 Ethilon. Hyphaema was noted at the end of the surgery. Intravitreal injection of vancomycin and amphotericin was given to prevent infection. Post-operative day 1 showed corneal haze inferiorly with hyphaema-as shown in Figure 3.

Post-operatively he developed cataract which self-absorbed over a period of 2 months. His vision with +10 was $6 / 12$ with normal fundus. The parents were counselled regarding the need for a secondary intraocular lens later, once the bag underwent fibrosis as the zonules were damaged inferiorly along the entire tract of the hook. No IOP or retina abnormalities were observed during the follow up.

\section{Discussion}

A barbed fish hook embedded in the eye is daunting to both patient and the ophthalmic surgeon. ${ }^{3}$ Large intraocular foreign bodies are associated with poor visual prognosis. ${ }^{4}$ Fish hook injuries to the eye can involve the eyelids and the anterior or posterior segments. Aiello et al. described four main techniques to remove the fish hook..$^{5}$ (a) The back-out or retrograde method-this usually causes excessive damage during the extraction of the hook; (b) snatch or string-yank technique, (c) advance-and-cut technique which is what we used in 


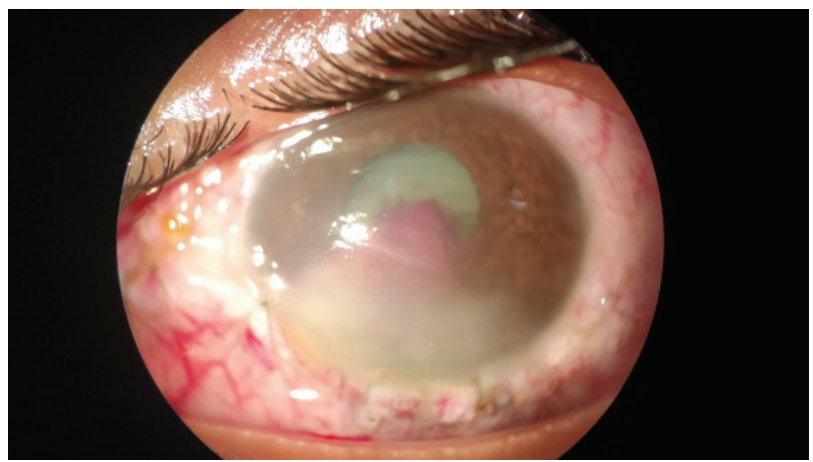

Fig. 3. Eye on post-operative day 1 showing hyphaema, traumatic cataract and inferior corneal haze.

our patient and (d) the needle-cover technique which is preferred for hooks that have penetrated the retina.

The manoeuvre of pushing the hook out through the eye is the same movement used in manipulating a curved suture needle. The shaft of the hook should be left long to facilitate manipulation. The extraocular barbs should be removed and this will require powerful steel cutters. This is called the advance-and-cut technique described by Ahmad et al. ${ }^{6}$ The use of cryotherapy in our patient helped minimize the bleed that would have occurred as the hook was imbedded at the limbus along the ciliary zone. The challenge in this case was the technique of removing the foreign body, and assistance from orthopaedic department for cutting the dense metallic knob at the entry site and also to minimize the bleeding as the hook was situated at the most vascular part of the eye. If the manoeuvring was not delicate, there was a chance that the choroid and retinal tissue would be damaged which would have given a poor visual outcome. The cryotherapy on table also reduced the risk of dense haemorrhage.

\section{Conclusion}

Management of ocular fish hook injuries can be daunting and very difficult for the ocular surgeon. These injuries often lead to poor visual prognosis; however, in some cases they may have a good long-term prognosis if prompt and appropriate surgical intervention is advocated. The advance-and-cut technique is a safe method to prevent damage to ocular tissues during removal of the hook. Cryotherapy adjacent to the shank of the hook in the scleral side prevented torrential bleed in this patient. The hook should be removed under careful examination using the correct technique. Also the shaft of the hook should be left long and no attempt should be made by non-trained personnel to remove it as doing so can 
result in further damage. Although many forms of ocular trauma are preventable, ocular trauma will continue to represent a significant problem in the foreseeable future. Education and common sense can effectively reduce the number of ocular injuries and ophthalmologists can and should play a key role in the education of patients. It is not enough to merely tell a patient to use some eye protection, but rather prescribe a specific appropriate protective eyewear

\section{References}

1. Cao H, Li L, Zhang M, Li H. Epidemiology of pediatric ocular trauma in the Chaoshan Region, China, 2001-2010. PLoS One. 2013;8:e60844.

2. Agrawal R, Laude A, Taneja M. Fish hook injury of the eye. Int Ophthalmol. 2012;32(3):269-271.

3. Bartholomew RS, MacDonald M. Fish hook injuries of the eye. Br J Ophthalmol. 1980;64:531-533.

4. Jonas JB, Knorr HL, Budde WM. Prognostic factors in ocular injuries caused by intraocular or retrobulbar foreign bodies. Ophthalmology. 2000;107(5):823-828.

5. Aiello LP, Iwamoto M, Guyer DR. Penetrating and ocular fish hook injuries: surgical management and long-term visual outcome. Ophthalmology. 1992;99(6):862-866.

6. Ahmad SS, Seng CW, Ghani SA, Lee JF. Cut it out technique for ocular fish-hook injury. J Emerg Trauma Shock. 2013;6(4):293-295. 